\title{
Study of the hadronization process in cold nuclear medium
}

\author{
N. Akopov, L. Grigoryan ${ }^{\text {a }}$, Z. Akopov \\ Yerevan Physics Institute, Br.Alikhanian 2, 375036 Yerevan, Armenia ${ }^{\text {b,c }}$
}

Received: 2 April 2010 / Revised: 4 August 2010 / Published online: 30 September 2010

(C) The Author(s) 2010. This article is published with open access at Springerlink.com

\begin{abstract}
The improved two-scale model is used to perform the fit to the semi-inclusive deep-inelastic scattering (SIDIS) data of HERMES experiment at DESY on nuclear targets. The ratio of hadron multiplicity on nuclear target to the deuterium one is chosen as observable, as usually. The two-parameter's fit gives satisfactory agreement with the data in term of $\chi^{2}$ criterium. Best values of parameters are then used to calculate the nuclear multiplicity ratio for the hadrons not included in the fit procedure.
\end{abstract}

\section{Introduction}

Hadronic reactions in a nuclear medium, either cold or hot can shed additional light on the hadronization process. Numerous measurements of hadron production on nuclear targets in SIDIS of leptons [1-8] are available. In ultra relativistic heavy-ion collisions the jet-quenching and parton energy-loss phenomena are observed $[9,10]$. In each case the observed hadron yields are differed from those in the corresponding reactions on free nucleons. In comparison with other reactions leptoproduction has the virtue that energy and momentum of the struck parton are well determined, as they are tagged by the scattered lepton. Study of hadron production in SIDIS on nuclear targets offers an opportunity to investigate the quark (string, color dipole) propagation in nuclear matter and the space-time evolution of the hadronization process. If the final hadron is formed inside the nucleus, it can interact via the relevant hadronic cross section, causing further reduction of the hadron yield. The perturbative QCD cannot describe hadronization process because of the essential role of "soft" interactions. Therefore, the understanding of this process on the phenomenological level is of basic importance for development of the theory. For this purpose we investigate the nuclear attenuation (NA),

\footnotetext{
a e-mail: leva@mail.desy.de

${ }^{b}$ N. Akopov supported by ISTC Grant A-1606.

${ }^{\mathrm{c}}$ L. Grigoryan supported by DESY, Deutsches Elektronen Synchrotron.
}

which is a ratio of differential hadron multiplicity on a nucleus to that on deuterium.

$R_{M}^{h}(v, z)=\frac{\left(\frac{N^{h}(v, z)}{N^{e}(v)}\right)_{A}}{\left(\frac{N^{h}(v, z)}{N^{e}(v)}\right)_{D}}$,

where $z=E_{h} / v, E_{h}$ and $v$ are energies of the final hadron and virtual photon respectively, $N^{h}(\nu, z)$ is the number of semi-inclusive hadrons at given $v$ and $z$ and $N^{e}(v)$ is the number of inclusive DIS leptons at given $v$. Subscripts $A$ $(D)$ denote that reaction takes place on nucleus (deuterium) respectively. In the above formula more variables like the photon virtuality- $Q^{2}$, transverse hadron momentum in respect to the virtual photon direction- $-p_{t}$, over which the NA is averaged, are not written. At present, several phenomenological models for description of the NA [11-30] are available. The simple version of the string model, so called TwoScale Model (TSM), was proposed by European Muon Collaboration for the description of its experimental data [2]. In Ref. [16] improved version of TSM (ITSM) was proposed. In present work ITSM is used to perform a fit to the recent SIDIS data of HERMES experiment on nuclear targets [7]. For a fit we use the more precise (high statistic) part of the data sample including one dimensional data for $\pi^{+}$and $\pi^{-}$mesons and two dimensional data for charged pions. One (two) dimensional data means that data are presented in form of function of one (two) variable. Then the $R_{M}^{h}$ for all measured hadrons were calculated with the values of parameters corresponding to the minimum values of reduced $\hat{\chi}^{2}=\chi^{2} /$ d.o.f. (here d.o.f. denotes "degree of freedom"). Then the results of such "best fit" were compared with the experimental data.

The remainder of the paper is organized as follows. In Sect. 2 we briefly remind about the ITSM. In Sect. 3 the part of data included in fit and some details of fitting procedure are presented. Results are discussed and compared both with the different versions of present fit and with our preceding one [16]. In Sect. 4 we compare results of the fit with experimental data and discuss them. Conclusions are given in Sect. 5 . 


\section{ITSM}

Basic formula for NA in TSM [2] is:

$$
\begin{aligned}
R_{A}= & \pi \int_{0}^{\infty} b d b \int_{-\infty}^{\infty} d x \rho(b, x) \\
& \times\left[1-\int_{x}^{\infty} d x^{\prime} \sigma^{\operatorname{str}}(\Delta x) \rho\left(b, x^{\prime}\right)\right]^{A-1},
\end{aligned}
$$

where $b$ is the impact parameter, $x$-longitudinal coordinate of the DIS point, $\rho(b, x)$-nuclear density function, $x^{\prime}$-longitudinal coordinate of the string-nucleon interaction point, $\sigma^{\text {str }}(\Delta x)$ - the string-nucleon cross section on distance $\Delta x=x^{\prime}-x$ from DIS point, $A$-atomic mass number. The above equation does not take into account the final state interactions (FSI) in deuterium. In this paper we use, following [16], more precise formula for the ratio of multiplicities $R_{M}^{h}=R_{A} / R_{D}$. The string model is based on the idea that after DIS the knocked out (anti)quark does not leave the nucleon remnant, but forms a string (color dipole) with the (anti)quark on the fast and the nucleon remnant on slow ends, while the color string itself consists of gluons. Its longitudinal size must be larger than the transverse one, but cannot be essentially larger than the hadronic size because of confinement. The string can break down into two strings according to the following scenarios. First, when the quarkantiquark pair from the color field of the string is produced; and second, when the color interaction between the string and the nucleon (lying on its trajectory) has happened (see for instance [12, 18]). In the "history" of the string there are two time scales which are of interest for us. They are the time scales connected with the production of the first constituent (anti)quark of the final hadron and interaction of its two constituents for the first time. These two scales are (see Fig. 1): $\tau_{c}\left(l_{c}\right)$-constituent formation time (length) ${ }^{1}$; and $\tau_{h}$ $\left(l_{h}\right)$-yo-yo formation time (length). The yo-yo formation means, that the colorless system with valence contents and quantum numbers of the final hadron is formed, but without its "sea" partons. In the two-dimensional string model which satisfy the following conditions: (i) quark-antiquark pairs arising from the vacuum do not have energy; (ii) energy loss of the leading quark on unit length (string tension) is constant (widely known example is the Lund model), there is a simple connection between $\tau_{h}$ and $\tau_{c}$

$\tau_{h}-\tau_{c}=z \nu / \kappa$

\footnotetext{
${ }^{1}$ In relativistic units $(\hbar=c=1$, where $\hbar=h / 2 \pi$ is the Plank reduced constant and c-speed of light) $\tau_{i}=l_{i}, i=c, h$ because partons and hadrons move with near light speeds.
}

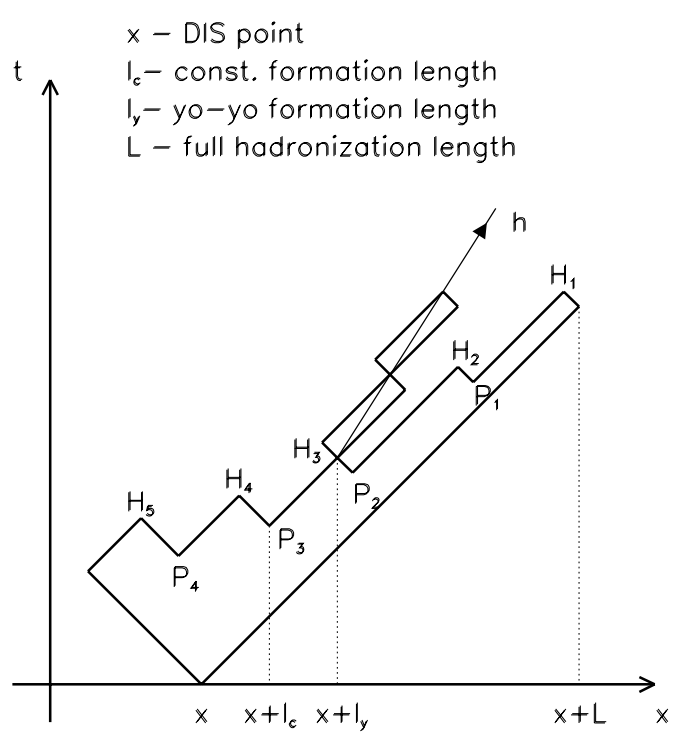

Fig. 1 Space-time structure of hadronization in the string model. The two constituents of the hadron are produced at different points. The first and second constituents of hadron $h$ are created at the points $\mathrm{P}_{3}$ and $\mathrm{P}_{2}$, respectively. They meet at $\mathrm{H}_{3}$ to form the hadron

where $\kappa$-string tension (string constant). Further we will use two different expressions for $\tau_{c}$. The first expression is obtained for hadrons containing leading quark [31]:

$\tau_{c}=(1-z) \nu / \kappa$.

The color string fully spends its energy on the distance of $L=v / \kappa$ beginning from the DIS point (see Fig. 1). Last hadron producing from the string is $h=H_{1}$, which contains leading quark and carries energy $E_{h}$. At distance $L$, the energy of the leading quark becomes equal to zero and whole energy of hadron is concentrated in another constituent. This constituent collects its energy from the string, and will have energy $E_{h}$ on distance $L$ only if it was produced on distance $E_{h} / \kappa=z \nu / \kappa$ from $L$. This is reflected in (3). It is important to note that the hadron produced on the fast end of string is not always necessarily the fastest hadron. Second expression for $\tau_{c}$ used in this paper is its average value:

$\tau_{c}=\int_{0}^{\infty} l d l D_{c}(L, z, l) / \int_{0}^{\infty} d l D_{c}(L, z, l)$,

where $D_{c}(L, z, l)$ is the distribution of the constituent formation length $l$ of summed over all ranks hadrons carrying momentum $z$. This distribution in framework of the standard Lund model [32] was obtained in Refs. [13, 33]:

$$
\begin{aligned}
& D_{c}(L, z, l) \\
&= L(1+C) \frac{l^{C}}{(l+z L)^{C+1}} \\
& \times\left(\delta(l-L+z L)+\frac{1+C}{l+z L}\right) \\
& \times \theta(l) \theta(L-z L-l),
\end{aligned}
$$


where $C=0.3$ is the parameter which controls the steepness of the standard Lund fragmentation function. The path traveled by the string between the DIS and interaction points is $\Delta x=x^{\prime}-x$. In the TSM the string-nucleon cross section has form:

$$
\begin{aligned}
\sigma^{\operatorname{str}}(\Delta x)= & \theta\left(\tau_{c}-\Delta x\right) \sigma_{q} \\
& +\theta\left(\tau_{h}-\Delta x\right) \theta\left(\Delta x-\tau_{c}\right) \sigma_{s}+\theta\left(\Delta x-\tau_{h}\right) \sigma_{h},
\end{aligned}
$$

where $\sigma_{q}, \sigma_{s}$ and $\sigma_{h}$ are the cross sections for interaction with the nucleon of the initial string, open string (the string containing first constituent (anti)quark of final hadron on its slow end) and final hadron, respectively. In this model the string-nucleon cross section is a function which jumps in points $\Delta x=\tau_{c}$ and $\tau_{h}$.

In reality the string-nucleon cross section starts to smoothly increase from the DIS point, and reaches the value of the hadron-nucleon one at $\Delta x=\tau$.

Unfortunately, it is impossible to obtain $\sigma^{\text {str }}$ from perturbative $\mathrm{QCD}$, at least in the region $\Delta x \sim \tau$. This means that some model for the shrinkage-expansion mechanism has to be introduced. In this work we use four versions of $\sigma^{\text {str }}$. Two of them having linear and quadratic dependence of the cross section on $\Delta x / \tau$, were taken from Ref. [35]. Let us briefly discuss the physical reason behind linear and quadratic dependence (see Ref. [36]). The QCD lattice calculations show that the confinement radius is much smaller than the mean hadronic radii. Consequently the color field in the hadrons is located in tubes with a transverse size much smaller than the longitudinal one. The valence quarks and diquarks are placed at the end-points of these tubes. In case of inelastic scattering, the interacting hadron-tubes intersect in the impact parameter plane. The probability of the crossing of the tubes is proportional to their length. This means that $\sigma^{\text {str }}$ increases proportional to $\Delta x / \tau$. In the naive parton model, the inelastic cross section of a hadron with a nucleon is proportional to the transverse area which is filled in by its partons, i.e. $\sigma^{\text {str }}$ increases proportional to $(\Delta x / \tau)^{2}$. The first version of $\sigma^{\text {str }}$ is based on quantum diffusion:

$$
\begin{aligned}
\sigma^{\operatorname{str}}(\Delta x)= & \theta(\tau-\Delta x)\left[\sigma_{q}+\left(\sigma_{h}-\sigma_{q}\right)\right. \\
& \times \Delta x / \tau]+\theta(\Delta x-\tau) \sigma_{h},
\end{aligned}
$$

where $\tau=\tau_{c}+c \Delta \tau, \Delta \tau=\tau_{h}-\tau_{c}$. We introduce the parameter $c(0<c<1)$ in order to take into account a well known fact, that the string starts to interact with hadronic cross section soon after creation of the first constituent quark of the final hadron and before creation of second constituent.

The second version follows from naive parton case:

$$
\begin{aligned}
\sigma^{\operatorname{str}}(\Delta x)= & \theta(\tau-\Delta x)\left[\sigma_{q}\right. \\
& \left.+\left(\sigma_{h}-\sigma_{q}\right)(\Delta x / \tau)^{2}\right]+\theta(\Delta x-\tau) \sigma_{h} .
\end{aligned}
$$

Two other expressions for $\sigma^{\text {str }}$ were also used [11, 14]:

$\sigma^{\mathrm{str}}(\Delta x)=\sigma_{h}-\left(\sigma_{h}-\sigma_{q}\right) \exp \left(-\frac{\Delta x}{\tau}\right)$

and:

$\sigma^{\operatorname{str}}(\Delta x)=\sigma_{h}-\left(\sigma_{h}-\sigma_{q}\right) \exp \left(-\left(\frac{\Delta x}{\tau}\right)^{2}\right)$.

One can easily note that at $\Delta x / \tau \ll 1$ the expressions (9) and (10) turn into (7) and (8), respectively. At the first glance it may seems that the ITSM, as opposed to the TSM, is actually a 1 -scale model. But one must note that $\tau$ is a function of two scales $\tau=(1-c) \tau_{c}+c \tau_{h}$ whereas the parameter $c$ regulates inclusion of each scale into $\tau$.

\section{Details of fit and results}

For the fit the semi-inclusive data [7] of HERMES experiment on four nuclear targets (helium, neon, krypton, xenon) and deuterium were used. Only most precise (high statistic) part of data was used. It was consisting from two pieces:

(i) the piece of the one dimensional data including the $v$ and $z$-dependences of $\pi^{+}$and $\pi^{-}$mesons. Each set consists from 9 experimental points, i.e. for this piece we have all together 144 points. The one dimensional data for nuclear multiplicity ratio are a functions of single variable $v$ or $z$, whereas in model, $R_{M}^{h}$ enters as a function of two variables $v$ and $z$ (the usage of two variables allows one to avoid the problem of additional integration over $z$ or $v$ in (1)). For this reason we introduce in the $R_{M}^{h}$, in case of one dimensional data, second variable by next way. In case of $v$-dependence, for each measured $v$ bin the value of $\hat{z}$ (averaged over the given $v$ bin), and in case of $z$-dependence, for each measured $z$ bin the value of $\hat{v}$ (averaged over the given $z$ bin) are taken from the experimental data;

(ii) the piece of the two dimensional data, containing the charged pions data on the same nuclei. This part is available in form of detailed binning over $v(z)$ and three slices over $z(v)$. We would like to remind that slices over $z$ are: first $0.2<z<0.4$, second $0.4<z<0.7$ and third $z>0.7$ and over $v$ : first $6<v<12 \mathrm{GeV}$, second $12<v<17 \mathrm{GeV}$ and third $17<v<23.5 \mathrm{GeV}$. Each slice of each dependence consists from 8 experimental points, besides third slices over $v$ in $z$-dependence, which consist from 7 experimental points, i.e. in this piece we have 188 points.

For one and two dimensional data we select all together 332 experimental points.

Now let us turn to the discussion of the ingredients of the string model. One of the important parameters is the string tension (string constant) which determines the energy loss by leading quark on unit length. In this work it was fixed at 
a static value determined by the Regge trajectory slope [3740]

$\kappa=1 /\left(2 \pi \alpha_{R}^{\prime}\right)=1 \mathrm{GeV} / \mathrm{fm}$.

For calculations the following nuclear density functions (NDF) were used. For deuterium the hard core deuteron wave functions from Ref. [41] were used. For ${ }^{4} \mathrm{He}$ the shell model [42] was used:

$\rho(r)=\rho_{0}\left(\frac{4}{A}+\frac{2}{3} \frac{(A-4)}{A} \frac{r^{2}}{r_{A}^{2}}\right) \exp \left(-\frac{r^{2}}{r_{A}^{2}}\right)$,

where $r_{A}=1.31 \mathrm{fm}$ for ${ }^{4} \mathrm{He}$. For ${ }^{20} \mathrm{Ne},{ }^{84} \mathrm{Kr}$ and ${ }^{131} \mathrm{Xe}$ the Woods-Saxon distribution was used

$\rho(r)=\rho_{0} /\left(1+\exp \left(\left(r-r_{A}\right) / a\right)\right)$.

Three sets of parameters for NDF's from (13) were used for the fit:

first set [43], $a=0.54 \mathrm{fm}$,

$r_{A}=\left(0.978+0.0206 A^{1 / 3}\right) A^{1 / 3} \mathrm{fm} ;$

second set $[33,34], a=0.54 \mathrm{fm}$,

$r_{A}=\left(1.19 A^{1 / 3}-\frac{1.61}{A^{1 / 3}}\right) \mathrm{fm} ;$

third set [44], $a=0.545 \mathrm{fm}$,

$r_{A}=1.14 A^{1 / 3} \mathrm{fm}$.

The corresponding values of $\rho_{0}$ were determined from the normalization condition:

$\int d^{3} r \rho(r)=1$.

Parameter $a$ is practically the same for all three sets, radius $r_{A}$ for the third set is larger by approximately $6 \%$ than the ones for the first and second sets. Let us briefly discuss the choice of the nuclear matter distribution functions. For deuterium the choice of the NDF is not important because the FSI are small. For light nucleus ${ }^{4} \mathrm{He}$ the shell model was used, because there was no alternative. For middle and heavy nuclei preferable NDF is Woods-Saxon distribution. However, there is some freedom in the choice of the parameters themselves, therefore we have included three sets of parameters (14)-(16), in order to study uncertainty of the fitting procedure related to the NDFs. Two expressions for $\tau_{c}$ were used for the fit-(3) and (4). For $\sigma^{\text {str }}(\Delta x)$ four different expressions from (7)-(10) were used. The values of $\sigma_{h}$ (hadron-nucleon inelastic cross section) used in the fit were set equal to: $\sigma_{\pi^{+}}=\sigma_{\pi^{-}}=20 \mathrm{mb}$. The same value of inelastic cross section was used for charged pions.
The fit was performed to tune two parameters: the initial value of string-nucleon cross section $\sigma_{q}$ and coefficient $c$.

The quantitative criterium $\hat{\chi}^{2}$ was used. As usually it was determined as:

$$
\begin{aligned}
\hat{\chi}^{2}= & \frac{1}{\left(n_{\exp }-n_{\text {par }}-1\right)} \\
& \times \sum_{n=1}^{n_{\exp }}\left(\frac{R_{M}^{h}(\text { theor })-R_{M}^{h}(\exp )}{\Delta R_{M}^{h}(\exp )}\right)^{2},
\end{aligned}
$$

where $n_{\text {exp }}$ and $n_{\text {par }}$ are numbers of experimental points and parameters; $R_{M}^{h}$ (theor) is the theoretical value for ratio at given point; $R_{M}^{h}$ (exp) and $\Delta R_{M}^{h}(\exp )$ are experimental values of $R_{M}^{h}$ and its error. Let us firstly discuss fit with use of total errors. Results are presented in the Tables 1 and 2. Easily to see that ITSM describes data on quantitative level. The version with $\tau_{c}(3)$ gives for $\hat{\chi}^{2}$ the values in order of $0.5-0.6$ for all versions of $\sigma^{\text {str }}$. Minimum $\hat{\chi}^{2}=0.50$ is obtained for $\sigma^{\mathrm{str}}(8)$ and NDF from (15) at values of parameters equal $\sigma_{q}=3.90 \pm 0.10 \mathrm{mb}$ and $c=0.161 \pm 0.009$. The version with $\tau_{c}(4)$ gives for $\hat{\chi}^{2}$ the values in order of unity. Here minimum $\hat{\chi}^{2}=0.67$ is obtained for $\sigma^{\text {str }}(10)$ and NDF from (15) at values of parameters equal $\sigma_{q}=1.99 \pm 0.14 \mathrm{mb}$

Table 1 Values of fitting parameters and $\hat{\chi}^{2}$ in case of $\tau_{c}(3)$ and total errors. For NDF from (13) versions 1, 2, 3 are sets of parameters

\begin{tabular}{|c|c|c|c|}
\hline \multicolumn{4}{|c|}{$\sigma^{\mathrm{str}}(7)$} \\
\hline $\mathrm{NDF}$ & $\sigma_{q} \mathrm{mb}$ & $c$ & $\hat{\chi}^{2}$ \\
\hline 1 & $0.62 \pm 0.15$ & $0.247 \pm 0.017$ & 0.63 \\
\hline 2 & $0.81 \pm 0.16$ & $0.231 \pm 0.019$ & 0.57 \\
\hline 3 & $0.91 \pm 0.16$ & $0.202 \pm 0.017$ & 0.61 \\
\hline \multicolumn{4}{|c|}{$\sigma^{\mathrm{str}}(8)$} \\
\hline $\mathrm{NDF}$ & $\sigma_{q} \mathrm{mb}$ & $c$ & $\hat{\chi}^{2}$ \\
\hline 1 & $3.71 \pm 0.10$ & $0.175 \pm 0.010$ & 0.55 \\
\hline 2 & $3.90 \pm 0.10$ & $0.161 \pm 0.009$ & 0.50 \\
\hline 3 & $4.12 \pm 0.12$ & $0.140 \pm 0.014$ & 0.57 \\
\hline \multicolumn{4}{|c|}{$\sigma^{\mathrm{str}}(9)$} \\
\hline $\mathrm{NDF}$ & $\sigma_{q} \mathrm{mb}$ & $c$ & $\hat{\chi}^{2}$ \\
\hline 1 & $1.29 \pm 0.12$ & $0.079 \pm 0.012$ & 0.55 \\
\hline 2 & $1.53 \pm 0.13$ & $0.065 \pm 0.012$ & 0.53 \\
\hline 3 & $1.76 \pm 0.14$ & $0.040 \pm 0.012$ & 0.59 \\
\hline \multicolumn{4}{|c|}{$\sigma^{\mathrm{str}}(10)$} \\
\hline $\mathrm{NDF}$ & $\sigma_{q} \mathrm{mb}$ & $c$ & $\hat{\chi}^{2}$ \\
\hline 1 & $3.91 \pm 0.10$ & $0.100 \pm 0.012$ & 0.56 \\
\hline 2 & $4.13 \pm 0.11$ & $0.087 \pm 0.012$ & 0.53 \\
\hline 3 & $4.39 \pm 0.12$ & $0.066 \pm 0.012$ & 0.60 \\
\hline
\end{tabular}
from (14), (15), (16), respectively. $\sigma^{\text {str }}(7)$ means $\sigma^{\text {str }}$ from (7) etc. 
Table 2 Values of fitting parameters and $\hat{\chi}^{2}$ in case of $\tau_{c}(4)$ and total errors

\begin{tabular}{|c|c|c|c|}
\hline \multicolumn{4}{|c|}{$\sigma^{\mathrm{str}}(7)$} \\
\hline $\mathrm{NDF}$ & $\sigma_{q} \mathrm{mb}$ & $c$ & $\frac{\hat{\chi}^{2}}{}$ \\
\hline 1 & $0.00 \pm 0.01$ & $0.372 \pm 0.012$ & 1.89 \\
\hline 2 & $0.00 \pm 0.01$ & $0.343 \pm 0.011$ & 1.71 \\
\hline 3 & $0.00 \pm 0.01$ & $0.313 \pm 0.012$ & 1.67 \\
\hline \multicolumn{4}{|c|}{$\sigma^{\text {str }}(8)$} \\
\hline $\mathrm{NDF}$ & $\sigma_{q} \mathrm{mb}$ & $c$ & $\hat{\chi}^{2}$ \\
\hline 1 & $1.27 \pm 0.11$ & $0.146 \pm 0.004$ & 1.01 \\
\hline 2 & $1.41 \pm 0.22$ & $0.137 \pm 0.018$ & 0.91 \\
\hline 3 & $1.28 \pm 0.23$ & $0.113 \pm 0.016$ & 0.92 \\
\hline \multicolumn{4}{|c|}{$\sigma^{\operatorname{str}}(9)$} \\
\hline NDF & $\sigma_{q} \mathrm{mb}$ & $c$ & $\hat{\chi}^{2}$ \\
\hline 1 & $0.00 \pm 0.01$ & $0.111 \pm 0.009$ & 0.97 \\
\hline 2 & $0.00 \pm 0.01$ & $0.085 \pm 0.009$ & 0.85 \\
\hline 3 & $0.00 \pm 0.01$ & $0.057 \pm 0.009$ & 0.84 \\
\hline \multicolumn{4}{|c|}{$\sigma^{\operatorname{str}}(10)$} \\
\hline $\mathrm{NDF}$ & $\sigma_{q} \mathrm{mb}$ & $c$ & $\hat{\chi}^{2}$ \\
\hline 1 & $1.82 \pm 0.14$ & $0.077 \pm 0.010$ & 0.74 \\
\hline 2 & $1.99 \pm 0.14$ & $0.068 \pm 0.010$ & 0.67 \\
\hline 3 & $2.03 \pm 0.16$ & $0.047 \pm 0.010$ & 0.71 \\
\hline
\end{tabular}

and $c=0.068 \pm 0.010$. We see, that although values of $\hat{\chi}^{2}$ in version of $\tau_{c}(4)$ approximately two times larger than for version $\tau_{c}(3)$, minimal values of $\hat{\chi}^{2}$ in two versions differ considerably smaller. Next observation is that in version with $\tau_{c}(3)$ (Table 1) local min of $\hat{\chi}^{2}$ for each choice of $\sigma^{\text {str }}$ corresponds to NDF from (15). In version with $\tau_{c}$ (4) (Table 2) for each choice of $\sigma^{\text {str }}$ we have approximately equal values of $\hat{\chi}^{2}$ for NDFs from (15) and (16). ${ }^{2}$ The common tendency is that $\sigma^{\text {str }}$ is essentially smaller than hadron-nucleon cross sections ( $\sigma^{\text {str }}$ in version of $\tau_{c}(4)$ considerably smaller than in version of $\left.\tau_{c}(3)\right)$ and coefficient $c$ essentially smaller than unity (the values of $c$ in the versions of $\tau_{c}(3)$ and $\tau_{c}(4)$ are approximately equal). The obtained values of $\hat{\chi}^{2}$ show, that total errors are still large and do not allow to verify finally the different versions of ITSM. Nevertheless, we will attempt to receive some conclusions from fit.

\footnotetext{
${ }^{2}$ Fit of version with $\tau_{c}(4)$ can depend from the standard Lund model parameter $C$. The widely using value of this parameter is $C=0.3$ [32]. For study the dependence of fit from this parameter we performed three parametric fit with $\tau_{c}(4), \sigma^{\mathrm{str}}(10)$, NDF from (15). As parameters serve $\sigma_{q}$, parameter $c$, and Lund parameter $C$, which in our fit can changes in limits $0.2 \leq C \leq 0.5$. In result of fit we obtained $\hat{\chi}^{2}=0.64$, $\sigma_{q}=2.166 \pm 0.141, c=0.069 \pm 0.010$ and $C=0.500 \pm 0.022$. This result means, that including in fit third parameter does not change basic result considerably, but only shift the value of parameter $C$.
}

Except the basic fit which was discussed above, three another fits were also done. First of them was performed with the same data set as basic one but with three free parameters instead two. Two of them were taken the same as in basic fit and as a third parameter the string tension $\kappa$ was used. The results of fit with $\tau_{c}(3)$ were obtained very close to the basic fit for both fitting parameters and $\hat{\chi}^{2}$. Indeed, for $\sigma^{\operatorname{str}}(8)$ and NDF in form of (15) the values $\sigma_{q}=3.87 \pm 0.042 \mathrm{mb}$, $c=0.18 \pm 0.01, \kappa=1.075 \pm 0.013 \mathrm{GeV} / \mathrm{fm}$ and $\hat{\chi}^{2}$ practically the same as basic were obtained. In this case inclusion of third parameter does not felt. The results of fit with $\tau_{c}(4)$ show the stronger dependence from the third parameter. For this case the following values for $\sigma^{\mathrm{str}}(10)$ and $\mathrm{NDF}(15)$ were obtained:

$\sigma_{q}=3.36 \pm 0.058 \mathrm{mb}, c=0.00 \pm 0.00, \kappa=0.73 \pm$ $0.008 \mathrm{GeV} / \mathrm{fm}$ and $\hat{\chi}^{2}=0.61$. It is easily to see that the values of parameters significantly differ from the corresponding values in basic fit, but $\hat{\chi}^{2}$ is close enough to the one for basic fit. Two other fits were performed separately for one and two dimensional pieces of data. Results are close to the ones for basic fit. When data are divided on one and two dimensional parts, the $\hat{\chi}^{2}$ for one dimensional data are obtained slightly larger than for two dimensional part because in first case statistical errors are smaller. But general situation is very resemble to the one for basic fit.

Let us compare the procedure and results of present fit with the our preceding fit [16]. At that time the SIDIS data of HERMES experiment for two targets: nitrogen [4] and krypton [5] were available. The $v$ - and $z$-dependences of $\pi^{+}$and $\pi^{-}$mesons with 58 available experimental points were included in the fit procedure. As the experimental errors only statistical ones were taken. Minimum values of $\hat{\chi}^{2}$ (best fit) for ITSM two parameter's fit were obtained for $\tau_{c}$ from (3) $\left(\hat{\chi}^{2}=1.4\right)$ and for $\tau_{c}$ from (4) $\left(\hat{\chi}^{2}=1.5\right)$. Unfortunately direct comparison of results of the preceding and the present fits is impossible. The quality of HERMES experiment data is increased essentially: (i) the present data have very small values of statistical errors (as a minimum two times smaller than of preceding data); (ii) in the preceding fit were included data for two nuclei only (nitrogen and krypton). The data on nitrogen had smaller number of experimental points and essentially larger errors than data on krypton, i.e. fit was mainly based on krypton data. In present case data are available for four nuclei from light to heavy (helium, neon, krypton and xenon) and these data have comparable statistics; (iii) as the statistical errors essentially decreased and now are on the level of systematic errors, for fit it is necessary use the total errors. Nevertheless we see that both fits point out, that the version with $\tau_{c}(3)$ describes data better than the version with $\tau_{c}(4)$. 


\section{Comparison with data and discussion}

The results of the performed fit are presented in Tables 1 and 2 . These tables contain the best values of the fitted parameters, their errors and $\hat{\chi}^{2}$ for the case of total experimental errors. In Figs. 2 and 3 the one dimensional data for the hadron multiplicity ratios $R_{M}^{h}$ for $\pi^{+}$and $\pi^{-}$mesons as a functions of variable $v$ (left panels) and $z$ (right panels) are presented. The results for ${ }^{4} \mathrm{He}$ (panels a and b), ${ }^{20} \mathrm{Ne}$ (c, d), ${ }^{84} \mathrm{Kr}(\mathrm{e}, \mathrm{f})$ and ${ }^{131} \mathrm{Xe}(\mathrm{g}, \mathrm{h})$ are presented. Experimental points were taken from Ref. [7] (filled circles). For comparison we presented in case of krypton previous HERMES data [5] (open circles) also. The theoretical curves were calculated with the best values of parameters obtained for the versions of model with constituent formation length in form of (3) (dashed curves) and (4) (solid curves). Version with $\tau_{c}$ in form of (3) describes data presented in Figs. 2 and 3 better than version with $\tau_{c}$ in form of (4), but size of presented figures does not allow to see this difference.

In Fig. 4 the two dimensional data for $R_{M}^{h}$ for charged pions on ${ }^{4} \mathrm{He}$ (panels a, b) and ${ }^{20} \mathrm{Ne}$ (panels c, d) as a functions of $v$ (left panels) and $z$ (right panels) are presented.

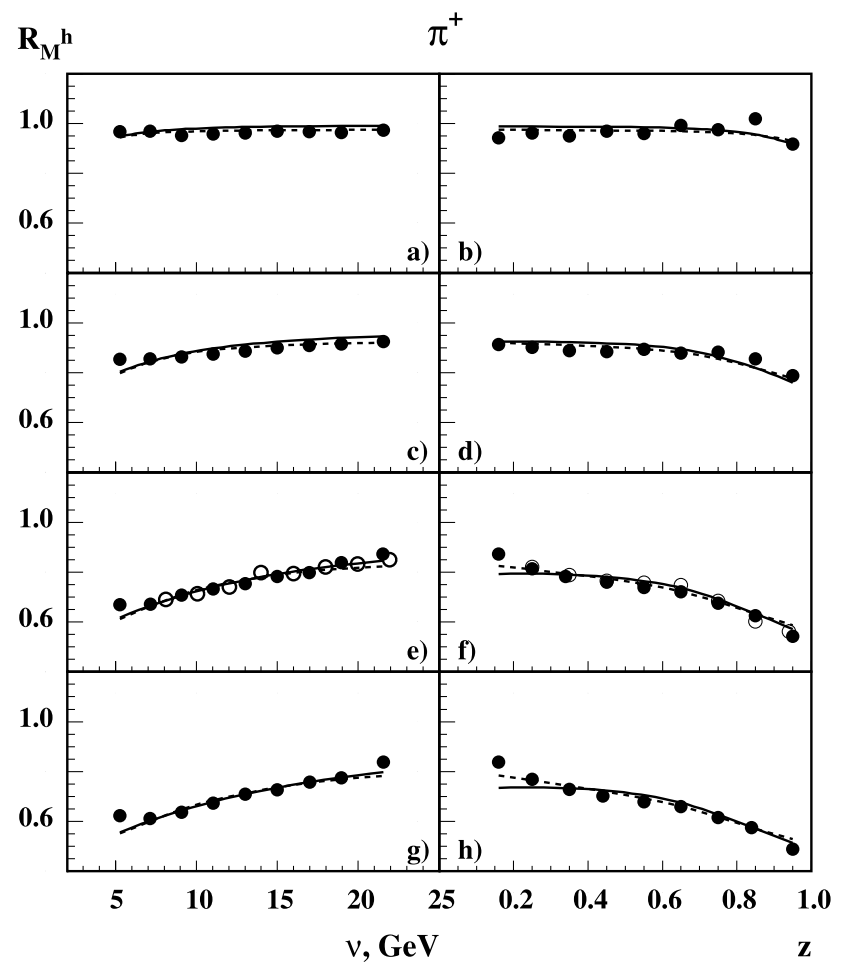

Fig. 2 One dimensional data. Hadron multiplicity ratio $R_{M}^{h}$ for $\pi^{+}$ mesons as a function of variable $v$ (left panels) and $z$ (right panels). The results are presented for ${ }^{4} \mathrm{He}(\mathbf{a}, \mathbf{b}),{ }^{20} \mathrm{Ne}(\mathbf{c}, \mathbf{d}),{ }^{84} \mathrm{Kr}(\mathbf{e}, \mathbf{f})$ and ${ }^{131} \mathrm{Xe}(\mathbf{g}, \mathbf{h})$. Experimental points from Ref. [7] (filled circles) and Ref. [5] (open circles). Statistical errors are presented. The curves were calculated with the best values of parameters obtained for the constituent formation length in form of (3) (dashed curves) and (4) (solid curves). These data were included in fit
In Fig. 5 the two dimensional data for $R_{M}^{h}$ for charged pions on ${ }^{84} \mathrm{Kr}$ (panels a, b) and ${ }^{131} \mathrm{Xe}$ (panels c, d) as a functions of $v$ (left panels) and $z$ (right panels) are presented. Experimental points are taken from Ref. [7]. Filled symbols are: triangles - experimental points for the first slice over $z$ (v) for $v-(z-)$ dependence; circles-for the second slice and stars-for the third slice. The open symbols represent the one dimensional data and were included in figures for comparison with two dimensional ones. The open circles were chosen for $\pi^{+}$and open triangles for $\pi^{-}$mesons. Unfortunately the two dimensional data for helium are not informative because the points from different slices are mixed. Data for ${ }^{20} \mathrm{Ne}$ are more useful because the separation of points from different slices partly takes place. The complete separation of data from different slices takes place for heavy nuclei (krypton and xenon). They carry additional information in comparison with one dimensional data and are important for the development of theoretical models. From these figures we see, that one dimensional data (open points) mainly coincide with second slices and do not reflect the behavior of data in first and third ones. The theoretical curves were calculated with the best values of parameters obtained for the constituent formation length in form of (3) (dashed curves) and (4) (solid curves). Let us remind that $\tau_{c}$ of (3) was obtained for leading hadron while $\tau_{c}$ of (4) is the average value of this quantity in the standard Lund model. As we already mentioned above the two dimensional data for helium pre-

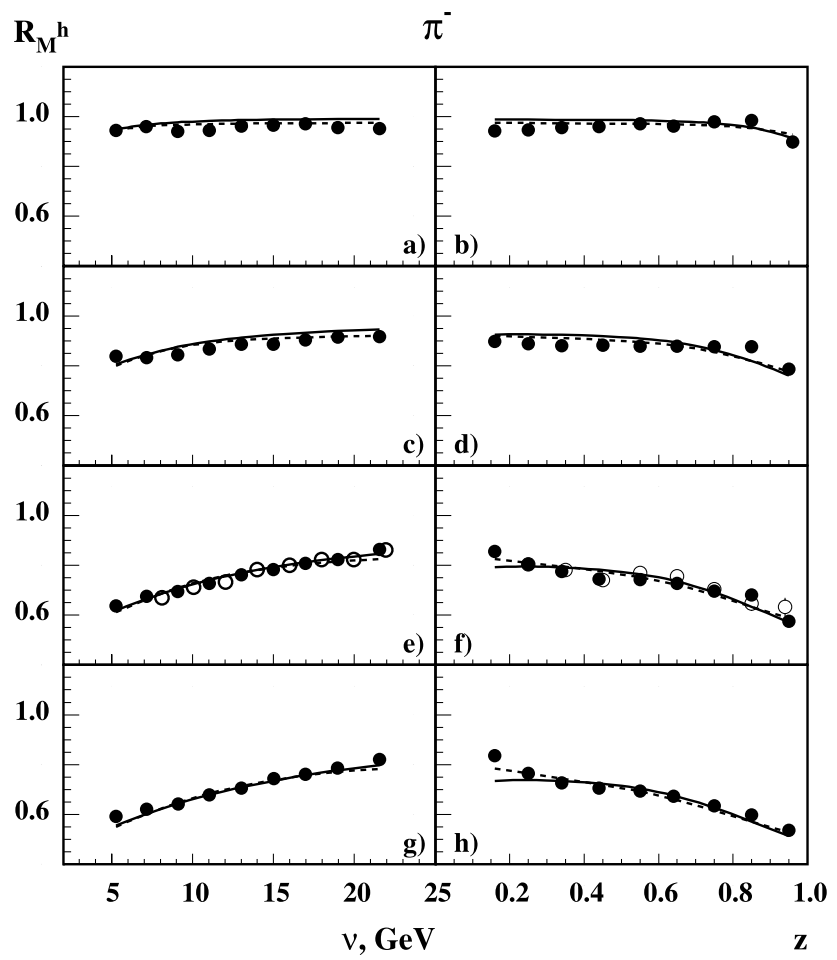

Fig. 3 The same as described in the caption of the Fig. 2 done for $\pi^{-}$ mesons. These data were included in fit 


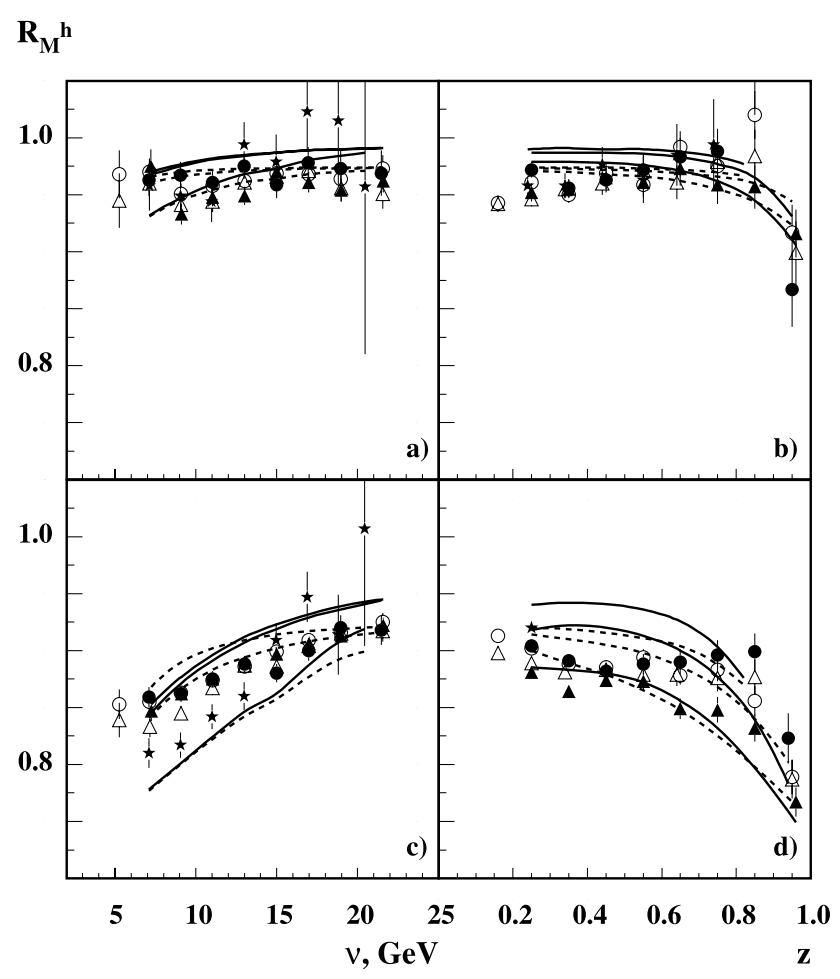

Fig. 4 The two dimensional data. The ratio $R_{M}^{h}$ for charged pions on ${ }^{4} \mathrm{He}$ (panels $\left.\mathbf{a}, \mathbf{b}\right)$ and ${ }^{20} \mathrm{Ne}$ (c, d) nuclei as a function of $v$ (left panels) and $z$ (right panels). Experimental points from Ref. [7]. Filled symbols are: triangles - for the first slice; circles - for second slice and stars - for third slice. Open symbols represent one dimensional data: circles - for $\pi^{+}$and triangles - for $\pi^{-}$mesons. The theoretical curves were calculated with the best values of parameters obtained for the constituent formation length in form of (3) (dashed curves) and (4) (solid curves). These data were included in fit

sented in Fig. 4 are less useful for comparison of different versions of model. We can state only, that theoretical curves do not contradict data. For middle nucleus (neon) presented in Fig. 4, despite on partial mixing of experimental points from different slices, we can state, that version with $\tau_{c}$ corresponding to (3) describes data better than one with $\tau_{c}$ corresponding to (4). And, at last, let us turn to the heavy nuclei (krypton and xenon) where situation is more clear. From Fig. 5 we see that in average the version with $\tau_{c}$ for leading hadron describes two dimensional data better than one from standard Lund model. The leading hadron approach satisfactory describes all three slices over $z$ for $v$-dependence (excepting last points), and also the first and second slices over $v$ for $z$-dependence but underestimates data in third slice, although the behavior is true. The main problem of the version of model with $\tau_{c}$ taken from standard Lund model (see (4)) is the unsatisfactory description of first and second slices over $z$ in $v$-dependence. This version of model practically does not differ data with small and middle $z$. It describes the $z$-dependence on satisfactory level excepting the region of

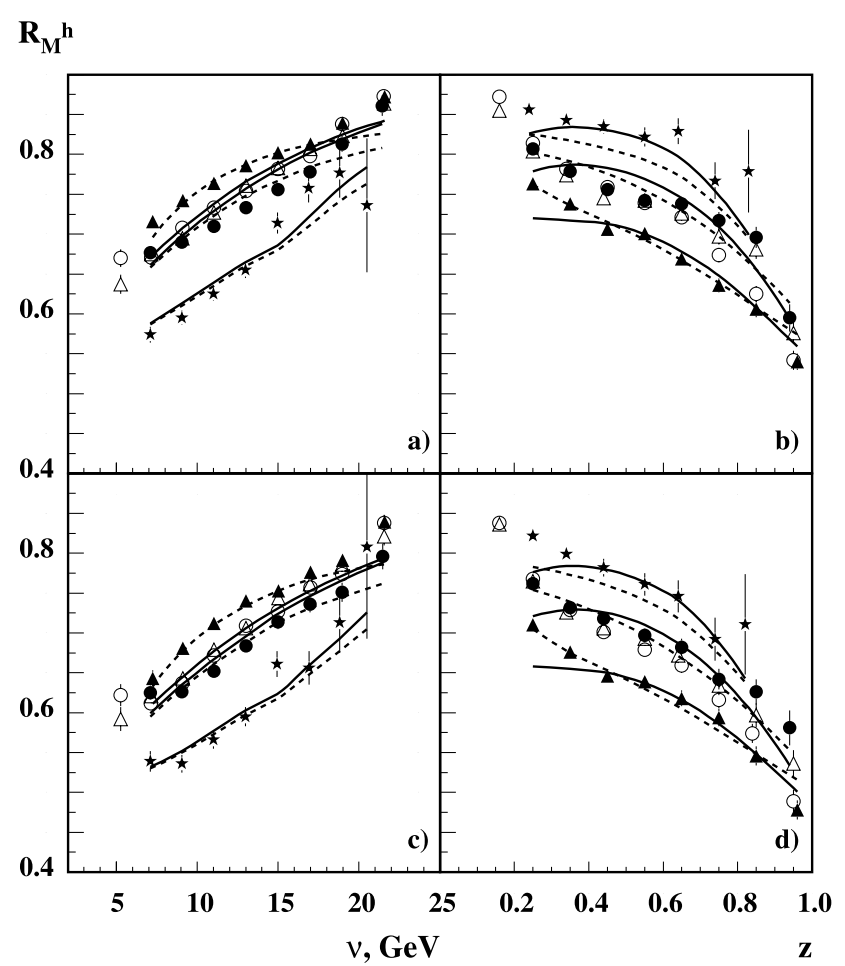

Fig. 5 The same as described in the caption of the Fig. 4 done for ${ }^{84} \mathrm{Kr}$ (panels $\mathbf{a}, \mathbf{b})$ and ${ }^{131} \mathrm{Xe}(\mathbf{c}, \mathbf{d})$ targets. These data were included in fit

small $z$. It is worth to mention that the difficulties in the description of $v$ - and $z$-dependences are mutually connected.

Furthermore, the NA for one dimensional data of hadrons produced on all nuclear targets (but not included in fit), were calculated in our model. In Figs. 6-9 the $v$ - and $z$-dependences are presented for $\pi^{0}, K^{+}, K^{-}$mesons and antiprotons, respectively. The hadron multiplicity ratio $R_{M}^{h}$ for mentioned hadrons as a function of variable $v$ (left panels) and $z$ (right panels) are presented for ${ }^{4} \mathrm{He}(\mathrm{a}, \mathrm{b}),{ }^{20} \mathrm{Ne}$ (c, d), ${ }^{84} \mathrm{Kr}$ (e, f) and ${ }^{131} \mathrm{Xe}(\mathrm{g}, \mathrm{h})$. Experimental points were taken from Ref. [7] (filled circles). For ${ }^{84} \mathrm{Kr}$ were taken also data from Ref. [5] (open circles). The theoretical curves were calculated with the best values of parameters obtained for the versions with $\tau_{c}$ in form of (3) (dashed curves) and (4) (solid curves). The following values of inelastic cross sections ${ }^{3} \sigma_{h}$ were used: $\sigma_{\pi^{0}}=\sigma_{K^{-}}=20 \mathrm{mb}$, $\sigma_{K^{+}}=14 \mathrm{mb}$ and $\sigma_{\bar{p}}=42 \mathrm{mb}$. Calculations, which were performed without additional fit, satisfactory describe data for $\pi^{0}$ (Fig. 6) and $K^{-}$(Fig. 8) mesons. Here we also have, as in case of $\pi^{+}$and $\pi^{-}$mesons, that version with $\tau_{c}$ in form of (3) describes data slightly better than with $\tau_{c}$ in form of (4).

It is worth to discuss data for $\mathrm{K}^{+}$mesons from Fig. 7 more in details, because here we have some problems. Let

\footnotetext{
${ }^{3}$ The hadron-nucleon inelastic cross sections using in this work can be found in Ref. [45].
} 


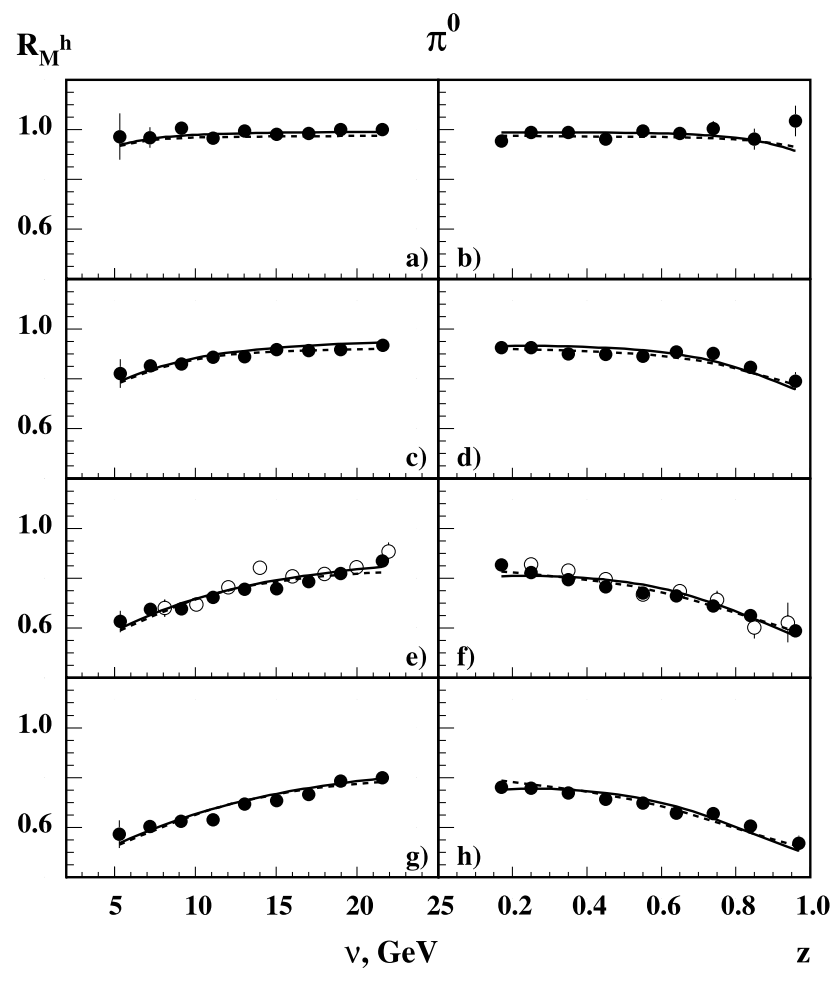

Fig. 6 One dimensional data. Hadron multiplicity ratio $R_{M}^{h}$ for $\pi^{0}$ mesons as a function of variable $v$ (left panels) and $z$ (right panels). The results are presented for ${ }^{4} \mathrm{He}(\mathbf{a}, \mathbf{b}),{ }^{20} \mathrm{Ne}(\mathbf{c}, \mathbf{d}),{ }^{84} \mathrm{Kr}(\mathbf{e}, \mathbf{f})$ and ${ }^{131} \mathrm{Xe}(\mathbf{g}, \mathbf{h})$. Experimental points were taken from Ref. [7] (filled circles) and Ref. [5] (open circles). The curves were calculated with the best values of parameters obtained for the constituent formation length in form of (3) (dashed curves) and (4) (solid curves). These data not included in fit

us begin with $v$-dependence. The data on ${ }^{4} \mathrm{He}$ are described very well. For ${ }^{20} \mathrm{Ne}$ nucleus we have disagreement between experimental data and theoretical model for the first two points. And, at last, for heavy nuclei we have essential underestimation of data by theoretical model. In this case comparison with the previous data for krypton helps to understand that problem rather in model than in data. In case of $z$-dependence we have satisfactory agreement for ${ }^{4} \mathrm{He}$ and ${ }^{20} \mathrm{Ne}$. Again description is worse for heavy nuclei. First three points are underestimated by model. We see that in region of small $z$ experimental values of $R_{M}^{h}$ decrease, while theoretical ones are rather constant. There is some disagreement between experimental data and theoretical model in case of antiprotons also (see Fig. 9). In this case theoretical curves slightly overestimate data.

As we mentioned above, data for $K^{+}$mesons and antiprotons did not include in fit. The theoretical curves were calculated with best values of parameters obtained from fit. Let us calculate the $\hat{\chi}^{2}$ for these cases with the fixed values of parameters obtained from fit. For $K^{+}$mesons we obtain for version $\tau_{c}(3) \hat{\chi}^{2}=6.36$ and for version $\tau_{c}(4)$ $\hat{\chi}^{2}=6.19$. These $\hat{\chi}^{2}$ are more than ten times larger than

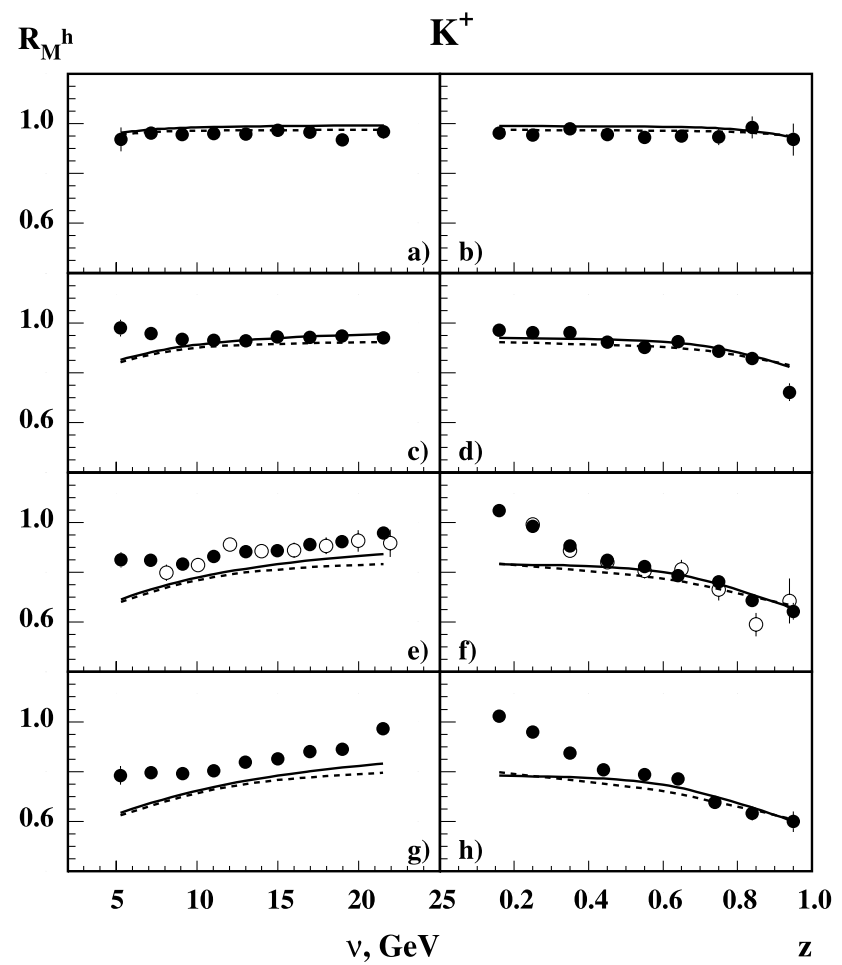

Fig. 7 The same as described in the caption of the Fig. 6 done for $K^{+}$ mesons

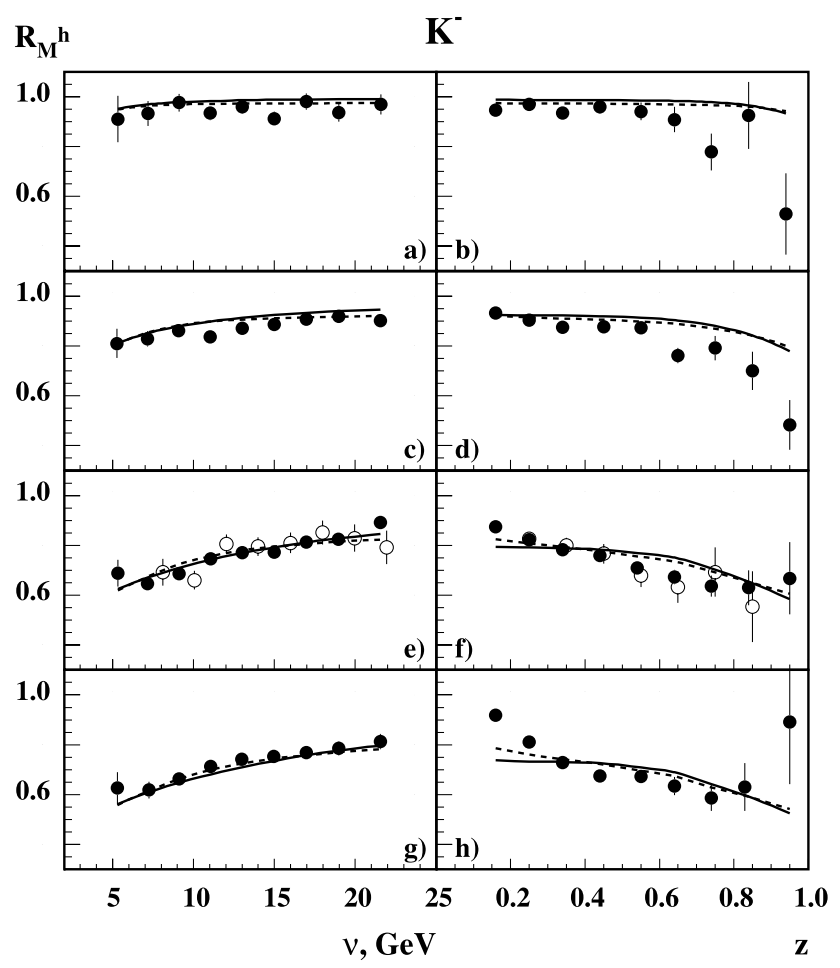

Fig. 8 The same as described in the caption of the Fig. 6 done for $\mathrm{K}^{-}$ mesons

the ones for basic fit. For the antiprotons on first glance situation better, because we have respectively 3.74 and 2.16. But this "improvement" is the result of larger errors only. 


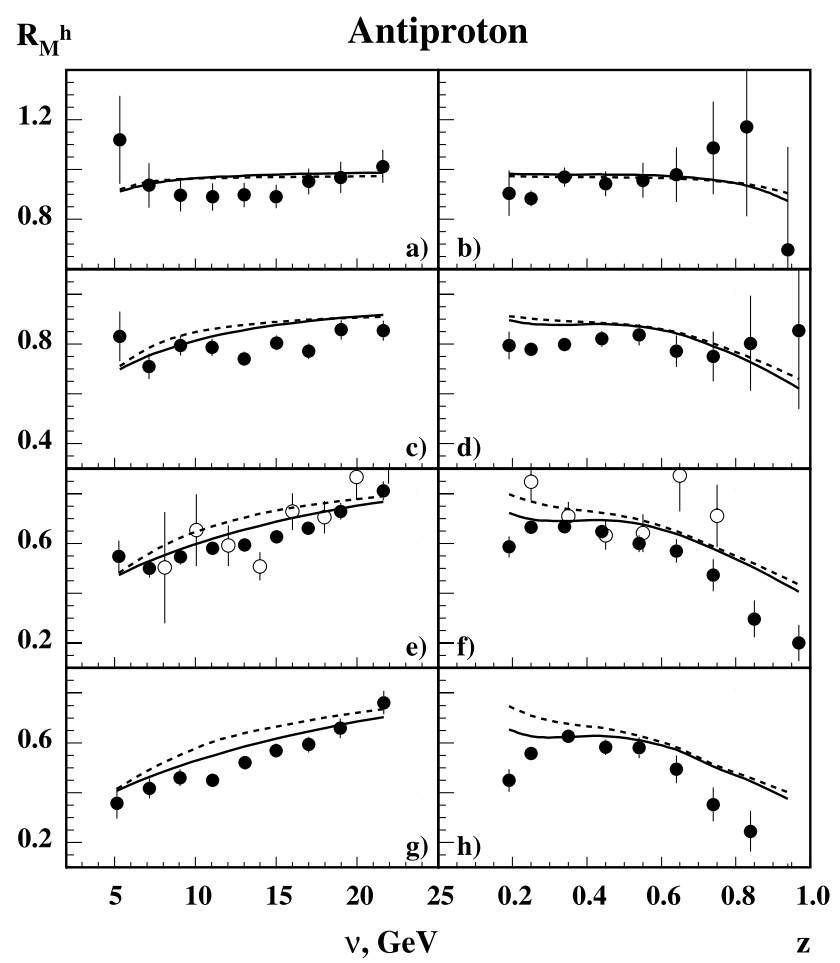

Fig. 9 The same as described in the caption of the Fig. 6 done for antiproton

In both cases the standard Lund model result is better than leading hadron approach one. The distribution of constituent formation lengths in the framework of the standard Lund model was obtained in work $[33,34]$ in supposition that only light quarks participate in fragmentation. In this approximation [32-34] the parameter $C=0.3$ arises (see (5) this paper). In this approach we can not distinguish the species of hadrons. Of course, it is rough approach for kaons and antiprotons. We can try to improve the result of standard Lund model by fitting parameter $C$ in limits $0.2 \leq C \leq 0.5$ at fixed values of other parameters. In result we obtained for $K^{+}$ mesons best value $\hat{\chi}^{2}=5.85$ at $C=0.5 \pm 0.009$ and for antiprotons $\hat{\chi}^{2}=2.07$ at $C=0.2 \pm 0.0165$. These values for $\hat{\chi}^{2}$ are close enough to the one for basic case $C=0.3$.

\section{Conclusions}

In this work the recent HERMES data [7] were used to perform the fit for ITSM. Two-parameter's fit demonstrates satisfactory agreement with data. The main goal was the further development of the model, in particular the choice of proper version for $\tau_{c}$. Minimum $\hat{\chi}^{2}$ (best fit) was obtained for version of model with $\tau_{c}$ in form of (3). The version with $\tau_{c}$ in form of (4) gave essentially larger value for $\hat{\chi}^{2}$. Comparison with the two dimensional data obtained, for the first time, by HERMES experiment, allowed to perform additional verification of the different choices of constituent formation length. In particular it was obtained, that version with $\tau_{c}$ in form of (3) has difficulty in description of third slice over $v$ in $z$-dependence, i.e. in region of large $v$. The difficulties of version with $\tau_{c}$ in form of (4) are more serious, because this version does not differ small and middle $z$ in $\nu$-dependence. Although version of model with $\tau_{c}$ in form of (3) describes data on the satisfactory level, the two dimensional data show, that we have some problem connected with the choice of the more adequate form for constituent formation length. More detail two dimensional data for identified hadrons that is expected from HERMES experiment will provide essentially better conditions for the choice of preferable version of the model in terms of different expressions for $\tau_{c}$ and, may be, $\sigma^{\text {str }}$. In all versions we have obtained that $\sigma_{q} \ll \sigma_{h}$. In our opinion it is indirect indication that at early stage of hadronization process the color transparency takes place. It is worth to mention that the same result was obtained in our preceding fit [16].

We do not include in consideration the NA of protons, because in this case additional mechanisms connected with color interaction (string-flip) and final hadron rescattering become essential (see for instance Refs. [12, 13]).

Open Access This article is distributed under the terms of the Creative Commons Attribution Noncommercial License which permits any noncommercial use, distribution, and reproduction in any medium, provided the original author(s) and source are credited.

\section{References}

1. L. Osborne et al., Phys. Rev. Lett. 40, 1624 (1978)

2. J. Ashman et al., Z. Phys. C 52, 1 (1991)

3. M. Adams et al., Phys. Rev. D 50, 1836 (1994)

4. A. Airapetian et al., Eur. Phys. J. C 20, 479 (2001)

5. A. Airapetian et al., Phys. Lett. B 577, 37-46 (2003)

6. A. Airapetian et al., Phys. Rev. Lett. 96, 162301 (2006)

7. A. Airapetian et al., Nucl. Phys. B 780, 1 (2007)

8. W. Brooks, H. Hakobyan, arXiv:0907.4606 [hep-ex]

9. K. Adcox et al., Phys. Rev. Lett. 88, 242301 (2002)

10. J. Adams et al., Phys. Rev. Lett. 92, 112301 (2004)

11. A. Bialas, Acta Phys. Pol. B 11, 475 (1980)

12. M. Gyulassy, M. Plumer, Nucl. Phys. B 346, 1 (1990)

13. J. Czyzewski, P. Sawicki, Z. Phys. C 56, 493 (1992)

14. R. Badalyan, Z. Phys. C 55, 647 (1992)

15. N. Akopov, G. Elbakian, L. Grigoryan, hep-ph/0205123 (2002)

16. N. Akopov, L. Grigoryan, Z. Akopov, Eur. Phys. J. C 44, 219 (2005)

17. N. Akopov, L. Grigoryan, Z. Akopov, Eur. Phys. J. C 49, 1015 (2007)

18. N. Akopov, L. Grigoryan, Z. Akopov, Eur. Phys. J. C 52, 893 (2007)

19. N. Akopov, L. Grigoryan, Z. Akopov, Phys. Rev. C 76, 065203 (2007)

20. N. Akopov, L. Grigoryan, Z. Akopov, arXiv:0810.4841 [hep-ph]

21. T. Falter, W. Cassing, K. Gallmeister, U. Mosel, Phys. Rev. C 70, 054609 (2004)

22. J. Dias De Deus, Phys. Lett. B 166, 98 (1986)

23. A. Accardi, V. Muccifora, H.J. Pirner, Nucl. Phys. A 720, 131 (2003) 
24. F. Arleo, Eur. Phys. J. C 30, 213 (2003)

25. X.-N. Wang, X. Guo, Nucl. Phys. A 696, 788 (2001)

26. E. Wang, X.-N. Wang, Phys. Rev. Lett. 89, 162301 (2002)

27. B. Kopeliovich, J. Nemchik, E. Predazzi, in Proceedings of the Workshop on Future Physics at HERA, ed. by G. Ingelman, A. De Roeck, R. Klanner. DESY, vol. 2, 1995/1996, p. 1038. nucl-th/9607036

28. B. Kopeliovich et al., hep-ph/0311220 (2003)

29. D.J. Dean et al., Phys. Rev. C 46, 2066 (1992)

30. R. Sassot, M. Stratmann, P. Zurita, arXiv:0906.5521 [hep-ph]

31. B. Kopeliovich, Phys. Lett. B 243, 141 (1990)

32. B. Andersson et al., Phys. Rep. 97, 31 (1983)

33. A. Bialas, M. Gyulassy, Nucl. Phys. B 291, 793 (1987)

34. T. Chmaj, Acta Phys. Pol. B 18, 1131 (1987)
35. G. Farrar et al., Phys. Rev. Lett. 61, 686 (1988)

36. B. Kopeliovich, J. Nemchik, L. Litov, Int. J. Mod. Phys. E 2, 767 (1993)

37. B. Kopeliovich, J. Nemchik, Preprint JINR E2-91-150 (1991)

38. B. Kopeliovich, J. Nemchik, Preprint of INFN-ISS 91/3 (1991), Roma

39. T. Sjostrand, L. Lonnblad, S. Mrenna, hep-ph/0108264 (2001)

40. T. Sjostrand, L. Lonnblad, S. Mrenna, LU TP 01-21

41. R.V. Reid, Ann. Phys. 50, 411 (1968)

42. L. Elton, Nuclear Sizes (Oxford University Press, Oxford, 1961), p. 34

43. A. Bialas et al., Phys. Lett. B 133, 241 (1983)

44. A. Capella et al., Phys. Rev. D 18, 3357 (1977)

45. C. Amsler et al., Phys. Lett. B 667, 1 (2008) 